\title{
Evaluation of the Disconnect between Hepatocyte and Microsome Intrinsic Clearance and In Vitro In Vivo Extrapolation Performance ${ }^{\text {氶 }}$
}

\author{
(1) Beth Williamson, Stephanie Harlfinger, and Dermot F. McGinnity \\ Drug Metabolism and Pharmacokinetics, Research and Early Development, Oncology R\&D, AstraZeneca, Cambridge, United \\ Kingdom
}

Received May 29, 2020; accepted August 17, 2020

\section{ABSTRACT}

The use of in vitro in vivo extrapolation (IVIVE) from human hepatocyte $(\mathrm{HH})$ and human liver microsome (HLM) stability assays is a widely accepted predictive methodology for human metabolic clearance (CLmet). However, a systematic underprediction of CLmet from both matrices appears to be universally apparent, which can be corrected for via an empirical regression offset. After physiological scaling, intrinsic clearance (CLint) for compounds metabolized via the same enzymatic pathway should be equivalent for both matrices. Compounds demonstrating significantly higher HLM CLint relative to $\mathrm{HH}$ CLint have been encountered, raising questions regarding how to predict CLmet for such compounds. Here, we determined the HLM:HH CLint ratio for 140 marketed drugs/compounds, compared this ratio as a function of physiochemical properties and drug metabolism enzyme dependence, and examined methodologies to predict CLmet from both matrices. The majority $(\mathbf{7 8} \%)$ of compounds displaying a high HLM:HH CLint ratio were CYP3A substrates. Using HH CLint for CYP3A substrates, the current IVIVE regression offset approach remains an appropriate strategy to predict CLmet (\% compounds overpredicted/correctly predicted/underpredicted 27/62/11, respectively).
However, using the same approach for HLM significantly overpredicts CLmet for CYP3A substrates (\% compounds overpredicted/correctly predicted/underpredicted 56/33/11, respectively), highlighting that a different IVIVE offset is required for CYP3A substrates using HLM. This work furthers the understanding of compound properties associated with a disproportionately high HLM:HH CLint ratio and outlines a successful IVIVE approach for such compounds.

\section{SIGNIFICANCE STATEMENT}

Oral drug discovery programs typically strive for low clearance compounds to ensure sufficient target engagement. Human liver microsomes and isolated human hepatocytes are used to optimize and predict human hepatic metabolic clearance. After physiological scaling, intrinsic clearance for compounds of the same metabolic pathway should be equivalent between matrices. However, a disconnect in intrinsic clearance is sometimes apparent. The work described attempts to further understand this phenomenon, and by achieving a mechanistic understanding, improvements in clearance predictions may be realized.

\section{Introduction}

For oral drug discovery programs, there is typically a requirement to design and develop low clearance (CL) compounds to ensure sufficient extent and duration of target engagement. Optimization of CL is usually one of the more significant challenges in drug discovery. With hepatic metabolic elimination remaining the predominant CL pathway for drugs (Cerny, 2016), the use of in vitro in vivo extrapolation (IVIVE) from metabolic stability assays is a widely accepted predictive methodology for human metabolic CL (CLmet) (Riley et al., 2005; Bowman and Benet, 2019a,b; Williamson et al., 2020). IVIVE comparisons of measured in vivo $\mathrm{CL}$ in animal species can assist with developing a cross-species mechanistic understanding of compound disposition.

All authors are AstraZeneca employees. https://doi.org/10.1124/dmd.120.000131.

S This article has supplemental material available at dmd.aspetjournals.org.
Moreover, for candidate drugs, successful prediction of an acceptable human in vivo CL is important to enable testing of the pharmacological hypothesis in the clinic and thus in reducing drug attrition for pharmacokinetics (PK) reasons (Hay et al., 2014; Davies et al., 2020).

Within drug discovery, two hepatic in vitro matrices are primarily used for metabolic stability assays to optimize and predict CLmet: human liver microsomes (HLM) and isolated human hepatocytes (HH). HLM offer the ability for enhanced-throughput intrinsic clearance (CLint) screening at relatively low cost and therefore can be used to triage suitable compounds into more expensive hepatocyte incubations. $\mathrm{HH}$ are regarded as the most predictive in vitro system since they contain the full complement of enzymes and transporters that a compound may encounter during first-pass metabolism, hence they often form the basis of IVIVE for CLmet. HLM and HH CLint, corrected for unbound fraction, can be scaled to in vivo CLint ( $\mathrm{ml} / \mathrm{min} / \mathrm{kg}$ ) using physiological parameters (Table 1). Therefore, with the reasonable assumption of

ABBREVIATIONS: A-B, apical to basolateral; AAFE, absolute AFE; AFE, average fold error; B-A, basolateral to apical; CL, clearance; CLint, intrinsic $\mathrm{CL}$; CLint,u, scaled unbound CLint; CLmet, metabolic CL; ER, efflux ratio; fuinc, fraction unbound in the incubation; $\mathrm{HH}$, human hepatocyte; $\mathrm{HLM}$, human liver microsome; ISEF, intersystem extrapolation factor; IVIVE, in vitro-in vivo extrapolation; LC, liquid chromatography; LogD, partition coefficient of a compound between octanol and water at pH7.4; MDCK, Madin-Darby Canine Kidney; MDR, multidrug resistance-1; MS, mass spectrometry; MW, molecular weight; Pgp, P-glycoprotein; PK, pharmacokinetics; Qh, hepatic blood flow; rCYP, recombinant CYP; RED, rapid equilibrium device; $\mathrm{RH}$, rat hepatocyte; UGT, uridine 5'-diphospho-glucuronosyltransferase; Vd, volume of distribution; WSM, "well stirred model”. 
TABLE 1

Human physiologic scaling factors

\begin{tabular}{cccccc}
\hline Species & $\begin{array}{c}\text { Liver Blood } \\
\text { Flow (Qh) } \\
(\mathrm{ml} / \mathrm{min} / \mathrm{kg})\end{array}$ & $\begin{array}{c}\text { Liver } \\
\text { Weight } \\
(\mathrm{g})\end{array}$ & $\begin{array}{c}\text { Body } \\
\text { Weight } \\
(\mathrm{kg})\end{array}$ & $\begin{array}{c}\text { Microsomal } \\
\text { Protein } \\
(\mathrm{mg}) / \mathrm{g} \text { Liver }\end{array}$ & $\begin{array}{c}\text { Hepatocellularity/g } \\
\text { Liver }\end{array}$ \\
\hline Human & 20.7 & 1500 & 70 & 40 & $120 \times 10^{6}$ \\
\hline
\end{tabular}

similar binding in the HLM and HH incubations and assuming the same metabolic pathways in both systems, the scaled HLM:HH CLint ratio should approximate 1 . Indeed, an indicator of the presence of significant additional metabolic pathways, such as glucuronidation, which is present in HH and not HLM (using only NADPH as a cofactor), would be a scaled HLM:HH CLint ratio significantly less than 1.

The commonly accepted approach for IVIVE involves inputting the CLint from HLM and HH into a mathematical model of liver perfusion, typically the "well stirred model" (WSM) (Rowland et al., 1973; Yang et al., 2007), to predict CLmet. However, this approach leads to a systematic underprediction of CLmet for reasons not presently understood (Riley et al., 2005; Foster et al., 2011; Bowman and Benet, 2019a). Many hypotheses to account for this underprediction have been proposed (Bowman and Benet, 2019a; Williamson et al., 2020), all of which should be considered during IVIVE for key compounds. However, none of these explanations solely satisfy the systematic nature of the CLmet underprediction. To account for this underprediction, an empirical correction can be applied to the in vitro data from hepatocytes or microsomes. This offset can be derived using a regression approach, in which the derived unbound in vitro and in vivo CLint values ( $\mathrm{ml} / \mathrm{min} / \mathrm{kg}$ ) form a correlation line from which future predictions of unbound in vivo CLint values for new compounds can be made (Riley et al., 2005; Sohlenius-Sternbeck et al., 2012). Notably, this offset appears to be relatively consistent between liver matrices, species, and laboratories and is independent of compound (Riley et al., 2005; Bowman and Benet, 2016; Wood et al., 2017).

Importantly, for CYP-mediated disposition, which remains the most prevalent CL pathway for drug-like compounds, there appears to be an overall comparable IVIVE predictive performance between HLM and HH (Riley et al., 2005; Chiba et al., 2009; Bowman and Benet, 2016). More recently, it has been postulated that underprediction from both matrices is increased as CL increases (Bowman and Benet, 2019a), and HLM are more accurate for predicting CL of CYP3A substrates (Bowman and Benet, 2019b).

Several reports have also noted a disconnect between HLM and HH CLint (Stringer et al., 2008; Foster et al., 2011; Bowman and Benet, 2019b), whereby a scaled HLM:HH CLint ratio significantly greater than 1 has been determined for specific compounds. Considerably larger CLint values in HLM relative to HH have been observed for CYP3A substrates yet, intriguingly, not for substrates of other drug metabolism enzymes (Bowman and Benet, 2019b). The mechanistic basis for this phenomenon has yet to be elucidated, although it has been hypothesized that this observation could be due to the overlapping substrate specificity between CYP3A and the efflux transporter, P-glycoprotein (Pgp), that is located on the hepatocyte membrane, restricting compound access to drug metabolism enzymes in hepatocytes relative to unhindered access to the same enzymes in microsomes (Bowman and Benet, 2019b), thus resulting in disproportionately lower CLint determined in $\mathrm{HH}$ relative to HLM.

Recently, Lombardo et al. (2018) published a large data set of compounds to investigate the relationship between physicochemical properties and human PK. This data set was used to investigate factors contributing to the phenomena, which we have observed in our laboratory, of the apparent disconnect between HLM and HH CLint and the predictive performance of IVIVE between matrices for such compounds. The data and analyses presented herein provide further insight regarding the HLM:HH CLint disconnect with a significant data set of 140 marketed drugs/compounds. By achieving a mechanistic understanding, improvements in IVIVE accuracy and CL predictions may be realized.

\section{Materials and Methods}

Compound Selection. The Lombardo data set (Lombardo et al., 2018) comprised 1352 compounds with measured human PK parameters and physicochemical properties. The data set was cross-referenced with compounds available in the AstraZeneca compound bank and filtered according to the following criteria:

- Compounds with a measured human CL approaching or less than liver blood flow (Qh $20.7 \mathrm{ml} / \mathrm{min} / \mathrm{kg}$ ) were included to disregard compounds with extraordinarily high CL that may have a significant extrahepatic component to their elimination.

- Only compounds with a molecular weight (MW) 150-800 and octanol: water partition coefficient $\log \mathrm{D}$ at $\mathrm{pH} 7.4(\operatorname{LogD}) 0.5-4$ were included to broadly represent the typical small-molecule physicochemical property space encountered in oral drug discovery.

- HLM CLint, HH CLint, human plasma protein binding, and incubational binding (fuinc) values were determined. Compounds with limits on measured values $(<$ and $>$ ) were subsequently excluded to avoid bias.

The resulting data set ( $n=140$ compounds) mainly comprised biopharmaceutics class 1 compounds predominantly cleared by hepatic metabolism, with human $\mathrm{CL}$ ranging $0.1-20 \mathrm{ml} / \mathrm{min} / \mathrm{kg}$. When required, additional data were generated, including Caco-2 permeability (Papp), Caco-2 efflux, Madin-Darby Canine Kidney (MDCK) multidrug resistance mutation 1 (MDR1) efflux, and CYP phenotyping (if the main route of elimination was via CYP, but the contribution of each isoform was unknown from literature sources). Given the reasonable assumption of consistencies in fuinc between species and matrices (Winiwarter et al., 2019), fuinc in HLM or rat hepatocytes (RH) was used. For $\log \mathrm{D}$ and fuinc, experimental values were supplemented with the use of in-house in silico models generated from machine learning methods. When possible, the main hepatic metabolic route of elimination was obtained from literature references or using databases, including the University of Washington Drug Interaction Database (this information was based on or an extract from Drug Interaction Database Copyright University of Washington, accessed: April 2020) (Supplemental Table 1). It was assumed that the clinical disposition pathway obtained from the literature reflects the main metabolic pathway in HH and HLM. Only in absence of such references were additional in vitro reaction phenotyping data obtained to define the main contributing enzyme (Supplemental Table 1).

For all in vitro assays detailed below, the experimental work was conducted at the Contract Research Organization, Pharmaron, China.

Materials. HLM (150 donors; Lot QQY and Lot 38289) were purchased from Corning or BioIVT (Shanghai, China), respectively. HH (10 donors; Lot LYB and Lot IRK) were purchased from BioIVT. Human plasma was purchased from BioIVT (mixed donors, with a minimum of two males and two females). Recombinant human CYP enzymes were purchased from CYPEX (Shanghai, China). EDTA-K2 was purchased from Beijing Chemical Reagents Company (Beijing, China). Bovine serum albumin was purchased from Beijing Xinjingke Biotechnology Co., Ltd (Beijing, China). FBS, Hank's balanced salt solution, nonessential amino acids, and the rapid equilibrium device (RED) were purchased from Gibco by Thermo Fisher Scientific (Shanghai, China). Dulbecco's modified Eagle's medium was purchased from Corning. 2-(N-morpholino)ethanesulfonic acid was purchased from Sigma (Shanghai, China). The 96-well equilibrium dialysis plate was purchased from LLC (CT). All other chemicals and materials were purchased from Solarbio S\&T Co., LTD (Beijing, China).

Determination of Human Hepatocyte CLint. Test compound was prepared to $10 \mathrm{mM}$ in $100 \%$ DMSO and further diluted to $100 \mu \mathrm{M}$ in $100 \%$ acetonitrile. The hepatocyte incubations were prepared in Leibovitz's L-15 Medium ( $\mathrm{pH} 7.4$ ) containing 1 million hepatocytes $/ \mathrm{ml}$ and a final compound concentration of $1 \mu \mathrm{M}$. Cell viability was determined using a Cellometer Vision, and $>80 \%$ cell viability 
was required to proceed with the compound incubation. The compound/cell solution $(250 \mu \mathrm{l})$ was incubated for 2 hours at $37^{\circ} \mathrm{C}$ and shaken at $900 \mathrm{rpm}$ on an Eppendorf Thermomixer Comfort plate shaker. Samples $(20 \mu 1)$ were taken at $0.5,5,15,30,45,60,80,100$, and 120 minutes and quenched with $100 \mu l$ of $100 \%$ ice-cold acetonitrile. Samples were shaken at $800 \mathrm{rpm}$ for 2 minutes and centrifuged at $4000 \mathrm{rpm}$ for 20 minutes at $4^{\circ} \mathrm{C}$ to pellet precipitated protein. The supernatant fraction was diluted 1:5 with deionized water, shaken at $1000 \mathrm{rpm}$ for 2 minutes, and further diluted 1:1 with deionized water. Samples were analyzed by liquid chromatography (LC)-mass spectrometry (MS)/MS.

As described previously (Williamson et al., 2020), submicromolar $\mathrm{K}_{\mathrm{m}}$ values (lower than compound assay concentrations) can impact CLint, but occurrence is infrequent, and determination of $\mathrm{K}_{\mathrm{m}}$ was beyond the scope of this work. Hence, $1 \mu \mathrm{M}$ was selected as an appropriate concentration in the CLint assays.

Determination of Human Microsome CLint. Test compound was prepared to $10 \mathrm{mM}$ in $100 \%$ DMSO and further diluted to $100 \mu \mathrm{M}$ in $100 \%$ acetonitrile. The microsomal incubations were prepared in phosphate buffered solution (pH 7.4) containing $1 \mathrm{mg} / \mathrm{ml}$ microsomal protein, $1 \mathrm{mM} \mathrm{NADPH}$, and a final compound concentration of $1 \mu \mathrm{M}$. After a preincubation with NADPH for 8 minutes, reactions were initiated through the addition of the test compound (final volume $250 \mu \mathrm{l}$ ) and incubated at $37^{\circ} \mathrm{C}$ in a water bath for 30 minutes. At each time point $(0.5,5,10,15,20,30$ minutes), $20 \mu \mathrm{l}$ of incubation mixture was quenched with $100 \mu \mathrm{l}$ of $100 \%$ ice-cold acetonitrile. Samples were shaken at $800 \mathrm{rpm}$ for 2 minutes and centrifuged at $4000 \mathrm{rpm}$ for 20 minutes at $4^{\circ} \mathrm{C}$ to pellet precipitated protein. The supernatant fraction was diluted 1:5 with deionized water, shaken at $1000 \mathrm{rpm}$ for 2 minutes, and further diluted 1:1 with deionized water. Samples were analyzed by LC-MS/MS.

Determination of Human Plasma Protein Binding. Plasma protein binding was completed using an RED device. Test compound was prepared to $1 \mathrm{mM}$ in $100 \%$ DMSO and further diluted in plasma to achieve a final compound concentration of $5 \mu \mathrm{M}$ in the incubation. Immediately, $50 \mu \mathrm{l}$ of the spiked plasma was aliquoted as a control $\mathrm{T}=0$ sample. The $\mathrm{T}=0$ sample was matrix-matched with $50 \mu \mathrm{l}$ of blank phosphate buffered solution (pH 7.4) and quenched with $400 \mu \mathrm{l}$ of $100 \%$ ice-cold acetonitrile. Phosphate buffered solution (pH 7.4; $500 \mu \mathrm{l}$ ) was added to the receiver chamber of the RED device, and spiked plasma $(300 \mu \mathrm{l})$ was added to the donor chamber. The plate was covered with a gaspermeable lid and incubated for 18 hours at $37^{\circ} \mathrm{C}$ with $5 \% \mathrm{CO}_{2}$ on an orbital shaker at $300 \mathrm{rpm}$. Remaining spiked plasma was incubated in a plastic plate for 18 hours at $37^{\circ} \mathrm{C}$ with $5 \% \mathrm{CO}_{2}$ on an orbital shaker at $300 \mathrm{rpm}$, representing an $\mathrm{T}$ $=18$ hours sample. At the end of incubation, $50 \mu \mathrm{l}$ of post dialysis sample from the donor or $\mathrm{T}=18$ sample and receiver wells were aliquoted into separate wells and matrix matched with $50 \mu \mathrm{l}$ of phosphate buffered solution ( $\mathrm{pH}$ 7.4) or blank plasma, respectively. The samples were subsequently quenched separately in $400 \mu \mathrm{l}$ of ice-cold $100 \%$ acetonitrile. Quenched samples, including $\mathrm{T}=0$, were shaken at $1000 \mathrm{rpm}$ for 10 minutes and centrifuged for 30 minutes at $4000 \mathrm{rpm}$ to pellet precipitated protein. The supernatant fraction was further diluted 1:1 with deionized water for analysis by LC-MS/MS. An eight-point calibration curve (1-7500 nM) matrix matched with plasma or phosphate buffered solution and quenched with $400 \mu \mathrm{l}$ of ice-cold $100 \%$ acetonitrile was used to determine the concentration in the donor and receiver wells. Compound recovery and stability in the plasma were determined using the $\mathrm{T}=0$ and $\mathrm{T}=18$ samples.

Determination of fuinc. HLM or RH binding was completed using a 96-well equilibrium dialysis plate. Test compound was prepared to $100 \mu \mathrm{M}$ in $100 \%$ DMSO and further diluted in $1 \mathrm{mg} / \mathrm{ml} \mathrm{HLM} /$ phosphate buffer or $1 \times 10^{6}$ inactivated (1 hour incubation with $1 \mathrm{mM}$ 1-Aminobenzotriazole and $1.5 \mathrm{mM}$ salicylamide) $\mathrm{RH} /$ phosphate buffer to achieve a final compound concentration of $1 \mu \mathrm{M}$ in the incubation. Immediately, $50 \mu \mathrm{l}$ of the spiked RH or HLM/phosphate buffered solution was aliquoted as a control $\mathrm{T}=0$ sample. The $\mathrm{T}=0$ sample was matrix matched with $50 \mu \mathrm{l}$ of blank phosphate buffered solution $(\mathrm{pH}$ 7.4) and quenched with $400 \mu \mathrm{l}$ of $100 \%$ ice-cold acetonitrile. Phosphate buffered solution $(\mathrm{pH} 7.4 ; 150 \mu \mathrm{l})$ was added to the receiver chamber of the dialysis block and spiked HLM/RH suspension $(150 \mu \mathrm{l})$ was added to the donor chamber. The plate was covered with a gas-permeable lid and incubated for 4 hours at $37^{\circ} \mathrm{C}$ with $5 \% \mathrm{CO}_{2}$ on an orbital shaker at $350 \mathrm{rpm}$. Remaining spiked matrix was incubated in a plastic plate for 18 hours at $37^{\circ} \mathrm{C}$ with $5 \% \mathrm{CO}_{2}$ on an orbital shaker at $300 \mathrm{rpm}$, representing an $\mathrm{T}=18$ hours sample. At the end of incubation, $50 \mu \mathrm{l}$ of postdialysis sample from the donor or $\mathrm{T}=18$ sample and receiver wells were matrix-matched with $50 \mu \mathrm{l}$ of phosphate buffered solution $(\mathrm{pH}$ 7.4) or blank $\mathrm{RH}$ or HLM/phosphate buffer, respectively. The samples were subsequently quenched separately in $400 \mu \mathrm{l}$ of ice-cold $100 \%$ acetonitrile. Quenched samples were shaken at $1000 \mathrm{rpm}$ for 10 minutes and centrifuged for 30 minutes at $4000 \mathrm{rpm}$ to pellet precipitated protein. The supernatant fraction was further diluted 1:1 with deionized water for analysis by LC-MS/MS. For RH fuinc, a fivepoint calibration curve (1-2000 nM) was generated, and for HLM fuinc, a sixpoint calibration curve $(1-2000 \mathrm{nM})$ was generated. Each standard was matrix matched with RH or HLM/phosphate buffer and phosphate buffered solution and quenched with $400 \mu \mathrm{l}$ of ice-cold $100 \%$ acetonitrile. The calibration curve was used to determine the concentration in the donor and receiver wells. Compound recovery and stability in the matrix were determined using the $\mathrm{T}=0$ and $\mathrm{T}=18$ samples.

Determination of Caco-2 or MDCK-MDR1 Permeability and Cell Efflux Ratio. Test compound was prepared to $10 \mathrm{mM}$ in $100 \%$ DMSO and further diluted to $200 \mu \mathrm{M}$ in $100 \%$ acetonitrile. Caco-2 and MDCK-MDR1 cells were diluted in Dulbecco's modified Eagle's medium (culture medium) at a density of $6.86 \times 10^{5}$ and $1.56 \times 10^{6}$ cells $/ \mathrm{ml}$, respectively. Fifty microliters of cell suspension was added to a 96-well transwell insert plate containing $50 \mu \mathrm{l}$ of culture medium. The base plate contained $25 \mathrm{ml}$ of culture medium. The Caco-2 cells were cultured for 14-18 days, and MDCK cells were cultured for 4-8 days, with culture medium replaced every other day. Once confluent (as determined by lucifer yellow) and electrical resistance was $>230 \Omega . \mathrm{cm}^{2}$ for Caco- 2 or $>42$ $\Omega . \mathrm{cm}^{2}$ for MDCK, the cells were used in the assay. The cells were washed and incubated with Hank's balanced salt solution containing 25 mM HEPES (pH 7.4) (transport buffer) for 30 minutes. Compounds were diluted to $2 \mathrm{mM}$ in $100 \% \mathrm{DMSO}$ and then further diluted to $10 \mu \mathrm{M}$ in transport buffer. To determine the rate of compound transport in the apical to basolateral (A-B) direction, $108 \mu \mathrm{l}$ of the $10 \mu \mathrm{M}$ compound solution was added to the transwell (apical), and $300 \mu \mathrm{l}$ of transport buffer was added to the receiver well (basolateral). Immediately, $8 \mu \mathrm{l}$ from the apical compound solution was diluted in $72 \mu \mathrm{l}$ of transport buffer and quenched with $240 \mu \mathrm{l}$ of ice-cold $100 \%$ acetonitrile; this sample represented a control $\mathrm{T}=0$ sample. Similarly, to determine the rate of compound transport in the basolateral to apical (B-A) direction, $308 \mu \mathrm{l}$ of the $10 \mu \mathrm{M}$ compound solution was added to the receiver well (basolateral), and $100 \mu \mathrm{l}$ of transport buffer was added to the transwell (apical). A T $=0$ sample was immediately prepared by diluting $8 \mu \mathrm{l}$ from the basolateral compound solution in $72 \mu \mathrm{l}$ of transport buffer and quenching with $240 \mu \mathrm{l}$ of ice-cold $100 \%$ acetonitrile. The plates were then incubated at $37^{\circ} \mathrm{C}$ for 2 hours. At the end of the 2 hour incubation, $8 \mu \mathrm{l}$ of sample was aliquot from the donor side (apical for A-B and basolateral for B-A) and added to $72 \mu 1$ of transport buffer and $240 \mu 1$ of ice-cold $100 \%$ acetonitrile. For the receiver compartments (basolateral for A-B and apical for B-A), $72 \mu \mathrm{l}$ was aliquoted and added to $240 \mu \mathrm{l}$ of ice-cold $100 \%$ acetonitrile. Quenched samples were shaken at $1000 \mathrm{rpm}$ for 5 minutes and centrifuged for 20 minutes at $4000 \mathrm{rpm}$ to pellet precipitated protein. The supernatant fraction was further diluted 1:1 with deionized water for analysis by LC-MS/MS.

CYP Reaction Phenotyping. Test compound was prepared to $10 \mathrm{mM}$ in $100 \%$ DMSO and further diluted to $200 \mu \mathrm{M}$ in $100 \%$ acetonitrile. Recombinant human CYP (rCYP: CYP3A4, CYP3A5, CYP2C9, CYP2C19, CYP2C8, CYP1A2, CYP2D6, CYP2E1, CYP2B6) were diluted with phosphate buffered solution ( $\mathrm{pH} 7.4$ ) to achieve a final concentration of $112 \mathrm{pmol} / \mathrm{ml}$. The diluted CYP solution was preincubated with test compound (final concentration $2 \mu \mathrm{M}$ ) at $37^{\circ} \mathrm{C}$ for 15 minutes. The reaction was initiated through the addition of the $10 \mathrm{mM}$ $\mathrm{NADPH}$ and incubated at $37^{\circ} \mathrm{C}$ on a plate shaker at $100 \mathrm{rpm}$ for 30 minutes. At each time point $(2.5,5,10,15,20,30$ minutes), $30 \mu \mathrm{l}$ of incubation mixture was quenched with $120 \mu \mathrm{l}$ of $100 \%$ ice-cold acetonitrile. Samples were shaken at $1000 \mathrm{rpm}$ for 10 minutes and centrifuged at $4000 \mathrm{rpm}$ for 20 minutes. The quench plate was further incubated at $4{ }^{\circ} \mathrm{C}$ for 30 minutes and recentrifuged at $4000 \mathrm{rpm}$ for 20 minutes to pellet precipitated protein. The supernatant fraction was diluted 1:1 with deionized water, shaken at $1000 \mathrm{rpm}$ for 2 minutes, and analyzed by LCMS/MS

LC-MS/MS Analysis. The MS/MS instrument used was either a Waters XEVO TQ-S, Waters XEVO TQ-D, or API 4000 (AB Sciex). The ultra-mass spectrometer used for sample analysis was completed in the multiple reaction monitoring mode (MS/MS). Reverse-phase high performance liquid chromatography with a $\mathrm{C} 18$ column was used to separate the analytes. A mobile phase of 99\% water/0.1\% formic acid (solvent A) and a solvent phase of 99\% acetonitrile/ $0.1 \%$ formic acid (solvent B) was used. A generic LC gradient elution was used at a flow rate of $0.5 \mathrm{ml} / \mathrm{min}$ with $95 \%$ solvent $\mathrm{A}$ and $5 \%$ solvent $\mathrm{B}$ for 0.3 minutes, after which the concentration of solvent B was increased to $95 \%$ over 0.9 minutes 
before restoring it back to $5 \%$ for the remaining 0.5 minutes. Mass-spectrometer methods were optimized for each compound.

For all assays, the $100 \%$ ice-cold acetonitrile used to quench the samples contained internal standards to ensure efficient extraction of sample, confirm injection into the mass spectrometer, and allow assessment of ionization variability. Data were accepted if the internal standard peak area coefficient of variation was $<20 \%$.

Data Analysis. The $t_{1 / 2}$ and, subsequently, the CLint of the compounds incubated in HH or HLM were calculated according to eqs. 1 and 2.

$$
\begin{gathered}
t_{\frac{1}{2}}(\min )=\frac{\operatorname{Ln}(2)}{- \text { slope }} . \\
C L_{\text {int }}\left(\mu \mathrm{l} / \mathrm{min} / \times 10^{6} \text { cellsormgprotein }\right)=\frac{\operatorname{Ln}(2) \times V}{t_{\frac{1}{2}}} .
\end{gathered}
$$

In which $\mathrm{V}\left(\mu \mathrm{l} / \mathrm{X} 10^{6}\right.$ cells or $\mathrm{mg}$ protein $)$ is the incubation volume $(\mu \mathrm{l})$ divided by the number of cells $\left(\mathrm{X}^{6} 0^{6}\right)$ or microsomal protein content $(\mathrm{mg})$ in the incubation.

The unbound fraction ( $\mathrm{fu}$ ) of the compounds in human plasma, HLM, or RH was calculated according to eq. 3 :

$$
\text { Fraction unbound }(f u)=\frac{[\text { Compound }]_{\text {Receiver }}}{[\text { Compound }]_{\text {Donor }}} .
$$

Compound recovery and stability in the relevant matrix were determined according to eqs. 4 and 5:

Compound recovery $(\%)=\frac{[\text { Compound }]_{\text {Receiver }}+[\text { Compound }]_{\text {Donor }}}{[\text { Compound }]_{T=0}} \times 100$.

$$
\text { Compound remaining at } 18 h(\%)=\frac{[\text { Compound }]_{T=18}}{[\text { Compound }]_{T=0}} \times 100 \text {. }
$$

The $t_{1 / 2}$ and, subsequently, the CLint of the compounds incubated with rCYP were determined as detailed in eqs. 6 and 7:

$$
\begin{gathered}
t_{\frac{1}{2} r \text { CYP }}(\min )=\frac{\operatorname{Ln}(2)}{- \text { slope }} . \\
C L_{\text {int }, r C Y P}(\mu \mathrm{l} / \mathrm{min} / \mathrm{pmol})=\frac{\operatorname{Ln}(2) \times V}{t_{\frac{1}{2}}} .
\end{gathered}
$$

In which, $\mathrm{V}(\mu \mathrm{l} / \mathrm{mg}$ protein) is the incubation volume divided by the $\mathrm{mg}$ of protein in the incubation.

The $\mathrm{CL}_{\text {int,rCYP }}$ was further scaled to account for CYP450 abundance and the intersystem extrapolation factors (ISEFs) utilizing the respective values incorporated in Simcyp (v19) using eq. 8:

$$
C L_{\text {int }, C Y P i}(\mu \mathrm{l} / \mathrm{min} / \mathrm{mg} \text { protein })=C L_{i n t, r C Y P i} \times C Y P_{i} \text { abundance } \times I S E F_{C Y P i} .
$$

In which CYP $\mathrm{P}_{\mathrm{i}}$ is the $i$ th CYP isoform tested out of $n$ CYP isoforms. $\mathrm{CL}_{\mathrm{int}, \mathrm{CYPi}}$ is the scaled CLint for the $i$ th CYP isoform, $\mathrm{CL}_{\text {int,rCYPi }}$ is the CLint determined for the $i$ th CYP isoform in $\mathrm{rCYP}(\mu \mathrm{l} / \mathrm{min} / \mathrm{pmol})$ (eq. 7), CYPi abundance is the abundance of the $i$ th CYP isoform in the HLM(pmol of cytochrome P450/mg protein), and ISEF $_{\mathrm{CYPi}}$ is the ISEF for the $i$ th CYP isoform.

The scaled $\mathrm{CL}_{\text {int,CYP }}$ values were summed to give the total scaled CLint in HLM, and the contribution of each CYP isoform in HLM was determined according to eq. 9 .

$$
\text { Contribution }_{C Y P i}(\%)=\frac{C L_{\text {int }, C Y P i}}{\sum_{i=1}^{n} C L_{i n t, C Y P i}} \times 100 .
$$

HLM:HH CLint ratio. Scaled HH and scaled HLM CLint values (m1/min/kg, eq. 10) were compared for each compound to calculate the difference, which was referred to as HLM:HH CLint ratio. Specifically, scaled HLM CLint ( $\mathrm{ml} / \mathrm{min} / \mathrm{kg})$ was divided by scaled HH CLint $(\mathrm{ml} / \mathrm{min} / \mathrm{kg})$. Assuming incubational binding was consistent between HLM and HH (Chen et al., 2017; Winiwarter et al., 2019), the difference in scaled CLint $(\mathrm{ml} / \mathrm{min} / \mathrm{kg})$ between the matrices was expected to be $\sim 1$ (based on the physiologic scaling factors noted in Table 1).

$$
\begin{aligned}
& \text { HLM or HH scaled CLint }(\mathrm{mL} / \mathrm{min} / \mathrm{kg}) \\
& \quad=[(\text { microsomal protein }(\mathrm{mg} / \mathrm{g} \text { liver }) \text { OR hepatocellularity } \\
& \left.\left(\times 10^{6} / \mathrm{g} \text { liver }\right)\right) \\
& \left.\times \frac{\text { liver weight }(\mathrm{g})}{\text { body weight }(\mathrm{kg})}\right] / 1000 .
\end{aligned}
$$

IVIVE. To compare in vitro hepatic CLint and in vivo CL for the 140 compound set, the WSM (eq. 11) (Rowland et al., 1973; Yang et al., 2007) was applied with a regression offset to correct for the observed systematic underprediction of in vivo $\mathrm{CL}$ :

$$
C \operatorname{Lmet}(\mathrm{ml} / \mathrm{min} / \mathrm{kg})=\frac{\left(Q_{h} \times f u \times C \operatorname{Lint}, u\right)}{\left(Q_{h}+f u \times C \operatorname{Lint}, u\right)} .
$$

Where, CLmet is the in vivo CL determined in plasma (assuming CL is hepatic metabolic), Qh is hepatic blood flow $(\mathrm{ml} / \mathrm{min} / \mathrm{kg}$ ), fu is the free fraction determined in plasma, and CLint, $\mathrm{u}$ is the scaled unbound intrinsic metabolic CL determined from $\mathrm{HH}$ or $\mathrm{HLM}(\mathrm{ml} / \mathrm{min} / \mathrm{kg})$.

\section{Regression Offset Approach.}

1. HH or HLM CLint values corrected for fuinc were scaled to the whole liver using physiological scaling factors (eq. 10; Table 1) to generate an in vitro CLint, u (units: $\mathrm{ml} / \mathrm{min} / \mathrm{kg}$ ).

2. In vivo CLint (units: $\mathrm{ml} / \mathrm{min} / \mathrm{kg}$ ) was back-calculated from human in vivo CLtotal values (in vivo CLint, $\mathrm{u} \mathrm{ml} / \mathrm{min} / \mathrm{kg}$ ), assuming hepatic metabolic CL and using the WSM (eq. 11) to deconvolute hepatic blood flow and fu in the blood (Yang et al., 2007).

3. Using a training set of 24 metabolically cleared drugs, the in vitro CLint,u and in vivo CLint,u values were compared for HH and HLM. A systematic underprediction of in vivo CLint,u from in vitro CLint,u was observed for both matrices. In our laboratory, the regression offset required to correct the underprediction was 3-fold for HLM and $\mathrm{HH}$ (Riley et al., 2005; Sohlenius-Sternbeck et al., 2012).

4. For the 140 compound set, the regression offset, previously defined as 3fold (see point 3 above), was applied prospectively to the in vitro CLint, u from $\mathrm{HH}$ and HLM and compared with the in vivo CLint, u values. If the CLint,u values (regression offset applied) for a compound were within 3 -fold of unity, CLmet was categorized as correctly predicted. Overpredictions and underpredictions of in vivo CLint, $\mathrm{u}$ were categorized as greater than 3-fold differences.

5. For scaling without the application of a regression offset, the in vitro CLint,u was calculated solely using the WSM (eq. 11).

Caco-2 and MDCK-MDR1 Papp (eq. 12) and, subsequently, efflux ratio were determined according to eq. 13 :

$$
\operatorname{Papp}\left(\times 10^{-6} \mathrm{~cm} / \mathrm{s}\right)=\frac{V_{A}}{\text { Area } \times \text { Time }} \times \frac{[\text { Compound }]_{\text {Acceptor }}}{[\text { Compound }]_{\text {Initial donor }}} .
$$

In which, $\mathrm{V}_{\mathrm{A}}$ is the volume in the acceptor well, Area is the surface area of the membrane, and Time is the total transport time.

$$
\text { Efflux ratio }=\frac{\operatorname{Papp}_{(B-A)}}{\operatorname{Papp}_{(A-B)}} .
$$

The average fold error (AFE) (eq. 14) and absolute average fold error (AAFE) (eq. 15) were calculated to determine the bias (AFE) and precision (AAFE) of the CL predictions:

$$
\begin{gathered}
A F E=10^{\frac{1}{x} \sum \log \left(\frac{\text { observed }}{\text { Precticed }}\right)} . \\
A A F E=10^{\frac{1}{N} \sum \log \left(\frac{\text { Obsered }}{\text { Predicted }}\right)} .
\end{gathered}
$$

HLM:HH CLint ratio data were not normally distributed. Therefore, a KruskalWallis with Dunn's multiple comparison correction was used to determine whether there was a difference in HLM:HH CLint ratio median between compounds for different classes (e.g., ion class, main metabolizing enzymes, etc.). To determine the Kruskal-Wallis statistic $(\mathrm{H})$ and the probability $(P)$, all data were pooled (ignoring the group from which the data belong) and ranked in ascending order. The rank sums were then combined to generate the $P$ value and 
a single statistic value termed $\mathrm{H}$. A large $\mathrm{H}$ refers to a large difference between rank sums. If the Kruskal-Wallis test was significant, a Dunn's multiple comparison was used to determine which groups were statistically different from each other by calculating a $P$ value (Mcdonald, 2014a; Dinno, 2015; Weaver et al., 2017).

A paired $\mathrm{T}$ test was used to compare HLM and HH CLint, u for uridine 5'-diphospho-glucuronosyltransferase (UGT), CYP1A, CYP2C, CYP2D6, and others because the variables were normally distributed, and a Wilcoxon matchedpairs signed rank test (Mcdonald, 2014b) was used to compare HLM and HH CLint,u for CYP3A because the variable was not normally distributed.

\section{Results}

HLM:HH CLint Ratio Dependencies. Assuming similar metabolic rates and routes, scaled HLM and HH CLint (in vitro CLint, $\mathrm{u} \mathrm{ml} / \mathrm{min} / \mathrm{kg}$ ) were expected to be equivalent (HLM:HH CLint ratio =1). In our data set, $51 \%$ of compounds had an HLM:HH CLint ratio $\sim 1$, with a maximum observed HLM:HH CLint ratio of 15 . The mean HLM: HH CLint ratio was 1.9, and the median was 1.1 for the 140 compound data set. Inherent experimental variability in the HLM and $\mathrm{HH}$ in vitro assays was assessed (unpublished data), and $\sim 95 \%$ of replicate CLint determinations for the same compound were within 2-fold. Thus, we categorized HLM:HH CLint ratio $\geq 2$-fold as a significant biologic difference.

The HLM:HH CLint ratio was significantly different between bases (mean 2.1) and acids (mean 1.0). However, the HLM:HH CLint ratio was not significantly different between neutrals and acids or neutrals and bases (Fig. 1A). HLM:HH CLint ratio was not correlated with MW, LogD, pKa, human Vd, or human CL (Fig. 1, B-F).

The dependence between the main metabolizing enzyme for compounds and HLM:HH CLint ratio was evaluated for the compound data set. The HLM:HH CLint ratio varied between metabolizing enzymes with the highest HLM:HH CLint ratio observed for CYP3A substrates (mean/median HLM:HH CLint ratio = 2.8/2.1) (Fig. 2; Supplemental Table 1). The difference in in vitro scaled CLint between HH and HLM was significantly different for CYP3A substrates (Wilcoxon matched pairs: $P<0.0001$ ). UGT substrates displayed a mean HLM:HH CLint ratio $>1$, but the median was 0.8 . For CYP2C, CYP2D6, and CYP1A substrates, the HLM:HH CLint ratio was $\sim 1$ (Fig. 2). The data set also contained 16 compounds (11\%, referred to as "Other" in Fig. 2) that were reported to be cleared via enzymes other than the major CYPs or UGTs, or the route was not defined, and the mean HLM:HH CLint ratio was 1.4. Interestingly, the vast majority (78\%) of compounds with an HLM:HH CLint ratio $\geq 2$ were CYP3A substrates. CYP3A HLM:HH CLint ratio was significantly different to the ratio determined for CYP2C, CYP2D6, and "other" substrates.

Overlapping substrate specificities between CYP3A and Pgp were investigated to understand whether efflux in $\mathrm{HH}$ provided an explanation for the high HLM:HH CLint ratio (Fig. 3). Caco-2 efflux ratio (ER) and MDCK-MDR1 ER were determined for 41 and 28 compounds, respectively, and represent compounds from all metabolizing enzyme families. For compounds with an ER $>2$ in Caco-2 and MDCK-MDR1 cells, the mean/median HLM:HH CLint ratio was 3.8/3.1 and 2.7/2.5, respectively. This was in contrast to the compounds with an $\mathrm{ER}<2$, which displayed mean/median HLM:HH CLint ratio of 1.3/1.0 and 1.6/ 1.1 in Caco-2 and MDCK-MDR1 cells, respectively. For compounds with an HLM:HH CLint ratio $\geq 2,50 \%$ and $64 \%$ of these compounds displayed an ER $>2$ in Caco-2 and MDCK-MDR1, respectively. For CYP3A substrates that had an HLM:HH CLint ratio $\geq 2,55 \%$ had an ER $>2$ in Caco-2 cells, and $67 \%$ had an ER $>2$ in MDCK-MDR1 cells.

In Vitro In Vivo Extrapolation. IVIVE accuracy of in vivo CLint, $\mathrm{u}$ was evaluated using both the regression offset approach and with no offset applied for HLM and HH as outlined in Materials and Methods.
When evaluating the set of 140 compounds as a whole, IVIVE performance using the regression offset showed minimal bias and good precision for both matrices. For HH, AFE was 1.3, AAFE was 2.9, and $\%$ compounds overpredicted/correctly predicted/underpredicted was 25/62/13, respectively, whereas for HLM, AFE was 1.6, AAFE 3.6, and $\%$ compounds overpredicted/correctly predicted/underpredicted was 34/52/14, respectively.

Prediction accuracy using the regression offset was comparable between HLM and HH when evaluating subcategories of UGT, CYP2D6, CYP2C, CYP1A, and "other" substrates (Figs. 4 and 5). However, using HLM and the regression offset approach for CYP3A substrates demonstrated a clear overprediction bias (AFE 3.1, AAFE 4.8, \% compounds overpredicted/correctly predicted/underpredicted 56/ 33/11, respectively) (Fig. 4E; Table 2). A comparison between CYP3A and non-CYP3A substrates using HLM and the regression offset approach is shown in Fig. 6. Conducting IVIVE using HLM without a regression offset for CYP3A substrates broadly corrected this overprediction bias (AFE 1.0, AAFE 3.2, \% compounds overpredicted/ correctly predicted/underpredicted 20/61/19, respectively, Fig. 6B; Table 2). However, using this approach (HLM without a regression offset) for the remaining non-CYP3A substrates led to a marked underprediction of in vivo CLint, u (AFE 0.3, AAFE 4.4, \% compounds overpredicted/correctly predicted/underpredicted 4/34/62, respectively, Fig. 6B; Table 2).

Using $\mathrm{HH}$ and the regression offset for CYP3A substrates demonstrated no clear bias (AFE 1.6, AAFE 3.1, \% compounds overpredicted/ correctly predicted/underpredicted 27/62/11, respectively) (Fig. 5E; Table 2). Likewise, a comparison between CYP3A and non-CYP3A substrates using $\mathrm{HH}$ showed no discernible difference in predictive performance (Fig. 6C; Table 2). It followed that conducting IVIVE using $\mathrm{HH}$ without a regression offset resulted in a significant underprediction bias for both CYP3A and non-CYP3A compounds (Table 2).

\section{Discussion}

To ensure sufficient target engagement, CL is often a key parameter to optimize before progression of oral candidate drugs into clinical development. With the majority of drugs eliminated via hepatic metabolic enzymes (Cerny, 2016), low CLint in HLM and HH is targeted, and the values are used to predict human metabolic CL. A systematic underprediction of in vivo CLint from both these hepatic matrices appears to be universally apparent (Riley et al., 2005; Sohlenius-Sternbeck et al., 2012; Bowman and Benet, 2019a), although it can be corrected for via a regression offset approach (Riley et al., 2005; Sohlenius-Sternbeck et al., 2012) and has been used prospectively to allow successful prediction of in vivo CL for many candidate drugs in our laboratory (Davies et al., 2020).

Typically, both HLM and HH are used in drug discovery. Once scaled, the HLM:HH CLint ratio should be $\sim 1$ for drugs cleared by the same drug metabolism enzyme pathways in both systems. However, significant differences between HLM and $\mathrm{HH}$ CLint have been highlighted by several groups (Stringer et al., 2008; Foster et al., 2011; Bowman and Benet, 2019b), and this "HLM:HH disconnect" has also been observed in our laboratory. When encountered in drug discovery, this HLM:HH disconnect phenomenon poses challenges; firstly to understand the reason(s) for differences in CLint for compounds cleared by the same enzymes and secondly to decide how to approach IVIVE for compounds that demonstrate this disconnect.

No correlation was observed between HLM:HH CLint ratio and human in vivo $\mathrm{CL}$, human $\mathrm{Vd}, \log \mathrm{D}, \mathrm{MW}$, or compound $\mathrm{pKa}$ for the 140 compound dataset (Fig. 1). The lack of correlation between HLM: $\mathrm{HH}$ CLint ratio and $\mathrm{pKa}$ is consistent with the hypothesis that the $\mathrm{pH}$ 

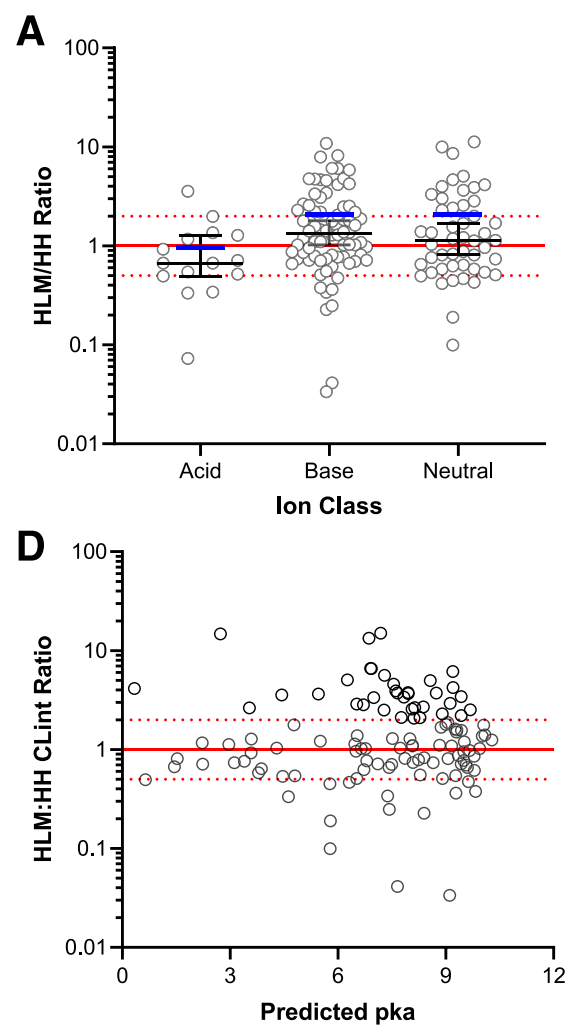


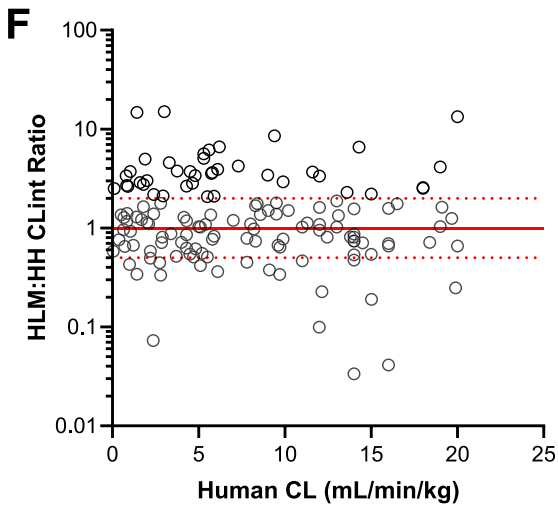

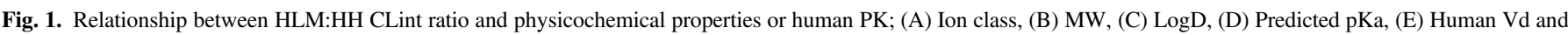

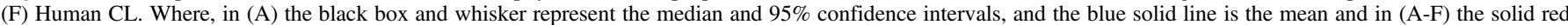

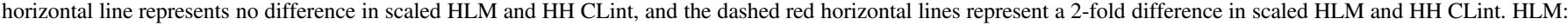

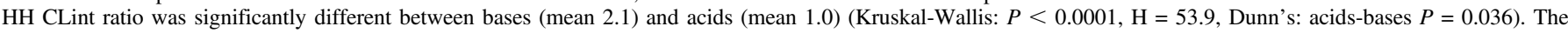
HLM:HH CLint ratio was not significantly different between acids and neutrals or neutrals and bases.

integrity of hepatocytes in suspension is destroyed (Berezhkovskiy, 2011), resulting in the same levels of ionized species present in hepatocyte and microsome assays conducted at $\mathrm{pH}$ 7.4. However, there was an observation that neutrals and bases (HLM:HH CLint ratio 2.1) demonstrate this HLM:HH disconnect more so than acids (HLM:HH CLint ratio 1.0), but this difference was only significant between acids and bases and would need more data specifically using acids to confirm. Another consideration is the potential for acids to be substrates of the hepatic organic anion transporting polypeptides (OATPs) present on the membrane of $\mathrm{HH}$. Active uptake into $\mathrm{HH}$ has the potential to become the rate-limiting step for the $\mathrm{CL}$ of these compounds. However, as shown previously (Di et al., 2012) and as further exemplified here, there was no difference in HLM:HH CLint ratio between OATP substrates [including paritaprevir, lesinurad, and repaglinide (Shebley et al., 2017; https://didb.druginteractionsolutions.org/drug/monograph/11166/\#maintransporters; https://didb.druginteractionsolutions.org/drug/monograph/ 1269/\#main-transporters)] and non-OATP substrates, suggesting there is no OATP dependence for the HLM:HH CLint ratio.

Building on the work by Bowman and Benet (2019b), the association of HLM:HH CLint ratio and metabolism via the major hepatic metabolic enzymes was assessed (Fig. 2). For CYP1A, CYP2C, and CYP2D6 substrates, the HLM:HH CLint ratio value was $\sim 1$, consistent with the CLint routes and rates being similar between matrices. Although UGT substrates displayed a high mean HLM:HH CLint ratio, this was influenced by two outliers (edaravone and mizolastine). The median HLM:HH CLint ratio was $0.8,<1$, as would be expected with the additional metabolism routes present in HH versus HLM. In agreement with Bowman and Benet (2019b), CYP3A substrates displayed a significantly higher CLint in HLM versus HH. These data conducted on such a large number of compounds confirm that this HLM:HH disconnect phenomenon is highly, if not exclusively, associated by cause or effect with CYP3A substrates. This would also explain the emerging trend that acids, which are not typically substrates of CYP3A, tend not to demonstrate this phenomenon. A thorough mechanistic understanding of the basis for the high HLM CLint relative to HH CLint and the explanation for the strong association of this phenomenon toward CYP3A substrates are clearly desirable. Efflux transporter activity in hepatocytes has been hypothesized to restrict compound access to metabolism enzymes in hepatocytes relative to unhindered access to the same enzymes in microsomes, thus leading to a high liver microsome: hepatocyte CLint ratio (Huang et al., 2010). Hence, overlapping substrate specificities between CYP3A and Pgp (Kim et al., 1999) may contribute to the observed HLM:HH CLint disconnect (Bowman and Benet, 2019b). We considered the relationship between ER and HLM:HH CLint ratio and determined that efflux transporter substrates displayed an HLM:HH disconnect (HLM:HH CLint ratio >2), whereas non-efflux substrates demonstrated an average HLM:HH CLint ratio of $\sim 1$, the theoretical expected ratio, irrespective of the cell line used to determine efflux potential (Fig. 3). These data suggest that an HLM:HH disconnect may be apparent for efflux substrates as a result of Pgp activity restricting CLint in $\mathrm{HH}$ relative to HLM. However, only $\sim 50 \%$ of CYP3A substrates that displayed an HLM:HH CLint ratio $\geq 2$ had an ER $>2$, as determined in either MDCK or Caco- 2 cells. Therefore, it would appear that the reason CYP3A substrates demonstrate this phenomenon as a class is not exclusively explained by Pgp activity. Additional confirmatory work would be beneficial to explore the dependence on HLM:HH CLint ratio with Pgp and other transporter activity. It should be noted that all efflux substrates in this 




Fig. 2. Evaluation of HLM:HH CLint ratio and the main metabolizing enzyme. Where, the black box and whisker solid lines represent the median and 95\% confidence intervals (CI), and the blue solid line is the mean. The solid red horizontal line represents no difference in scaled HLM and HH CLint, and the dashed red horizontal lines represent a 2-fold difference in scaled HLM and HH CLint. CYP3A HLM:HH CLint ratio was significantly different to CYP2C, CYP2D6, and Other (Kruskal-Wallis $P<0.0001, \mathrm{H}=29.4$, Dunn's: $P=0.0006$, $P=0.009$, and $P=0.03$, respectively)

data set (when measured) were highly permeable in the Caco-2 assay, suggesting passive permeability across a cell membrane was not a ratelimiting factor in hepatocytes. Furthermore, no correlation was observed between HLM:HH CLint ratio and passive permeability (unpublished data). There may be further additional contributory factors other than transporter activity that may lead to high HLM:HH CLint ratio, for example, whether the high HLM:HH CLint for CYP3A substrates is associated with cryopreserved and not freshly prepared HH. Hepatocyte cryopreservation would have to specifically decrease CYP3A isoform activity, possibly because of conformational changes in the enzyme or via affecting cofactor levels. Considering the high proportion of candidate drugs that are CYP3A substrates, more work in our laboratory and others is warranted.

Although the use of a regression offset approach to correct the widely reported systematic underprediction of in vivo CLint is a pragmatic solution to predict CL, it is not mechanistically satisfactory given the empirical nature of this approach. Similar to previous reports, from our laboratory and others (Riley et al., 2005; Sohlenius-Sternbeck et al., 2012), IVIVE predictive capability utilizing the regression offset approach for the whole dataset of compounds was broadly similar between $\mathrm{HH}$ and HLM; $62 \%$ and $52 \%$ of compounds predicted in vivo CLint,u within 3-fold from HH (AFE 1.3, AAFE 2.9) and HLM (AFE 1.6, AAFE 3.6).

Approximately $80 \%$ of compounds in this data set with an HLM:HH CLint ratio $\geq 2$ were CYP3A substrates, and likewise, our in-house experience with propriety AstraZeneca compounds demonstrates this phenomenon being strongly associated with CYP3A substrates. Given this strong association and the preponderance of candidate drugs metabolized primarily by CYP3A, our analysis focused on comparing and contrasting IVIVE performance for CYP3A versus non-CYP3A substrates. It was observed that when using HLM with an IVIVE regression offset approach for CYP3A substrates (Figs. 4E and 6A; Table 2), there was an overprediction ( $>3$-fold) of in vivo CLint, $\mathrm{u}$ (AFE 3.1 ) for $56 \%$ compounds, with only $33 \%$ correctly predicted. Not using the regression offset corrected this overprediction so that AFE approached 1, and the number of CYP3A substrates overpredicted reduced from $56 \%$ to $20 \%$, and those correctly predicted increased from $33 \%$ to $61 \%$ (Fig. 6B; Table 2). These data are consistent with previous reports for CYP3A substrates, which employed IVIVE without the use of a regression offset and demonstrated good IVIVE accuracy from HLM (Bowman and Benet, 2019b). However, using HLM without a regression offset for non-CYP3A substrates led to a marked underprediction of in vivo CLint,u (AFE 0.3) for $62 \%$ compounds, with $34 \%$ correctly predicted (Fig. 6B; Table 2 ). These data highlight that when using HLM for IVIVE, a different regression offset factor is optimal for scaling CYP3A substrates versus non-CYP3A substrates. A full mechanistic understanding is not immediately apparent, but the observation of both CYP isoform and matrix dependence on IVIVE methodology to predict CLmet may help to delineate the underlying reasons in the future. Based on these analyses, it is important to carefully consider IVIVE approaches so that CL is appropriately predicted from HLM data for each substrate class (Riley et al., 2005; Chiba et al., 2009; Bowman and Benet, 2016).

In contrast, for $\mathrm{HH}$, no prediction bias using the IVIVE regression offset approach existed for all categories of compound, including CYP3A substrates, with $62 \%$ of such compounds correctly predicted (AFE 1.6, AAFE 3.1) (Fig. 6C; Table 2). Overall, using HH, IVIVE performance using the regression offset approach for the whole data set showed minimal bias and good precision (AFE 1.3, AAFE 2.8) and correctly predicted CLint,u within 3-fold for $62 \%$ of compounds, a comparable performance to CYP3A substrates.
A

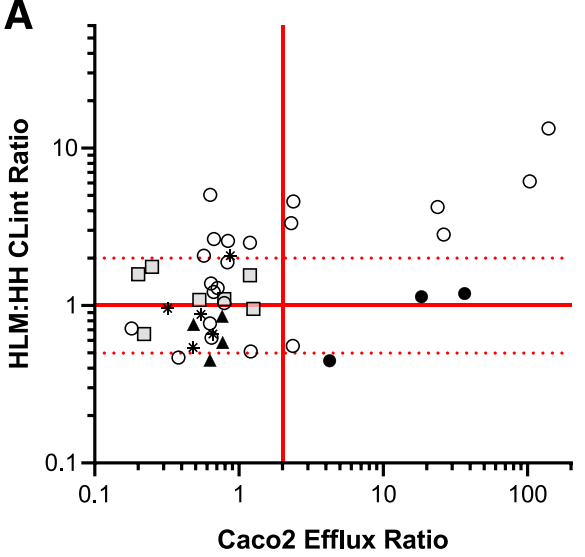

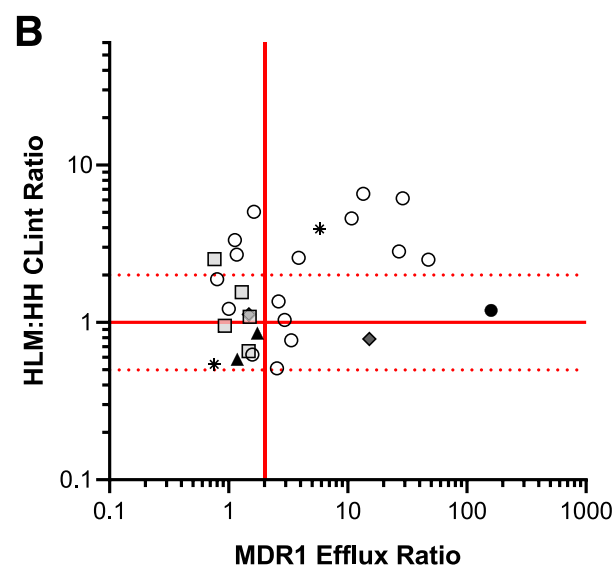

Fig. 3. HLM:HH CLint ratio compared with ER determined in (A) Caco-2 cells and (B) MDCK-MDR1 cells. Where, the solid red horizontal line represents no difference in scaled HLM and HH CLint, the dashed red horizontal lines represent a 2-fold difference in scaled HLM and HH CLint, and the solid red vertical line represents the efflux transporter substrate categorization; $\geq 2$ = efflux substrate, $<2=$ not an efflux substrate. Symbols represent the main metab-

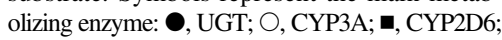
$\boldsymbol{\Delta}$, CYP2C; $\diamond$ CYP1A; and *, Other. In Caco-2, the number of compounds from each metabolizing enzyme group was: UGT $n=3$, CYP3A $n=22$, CYP2D $n=7$, CYP2C $n=4$, CYP1A $n=0$, and Other $n=5$. In MDCK-MDR1, UGT $n=1$, CYP3A $n=16$, CYP2D6 $n=5$, CYP2C $n=2$, CYP1A $n=2$, and Other $n=2$. 

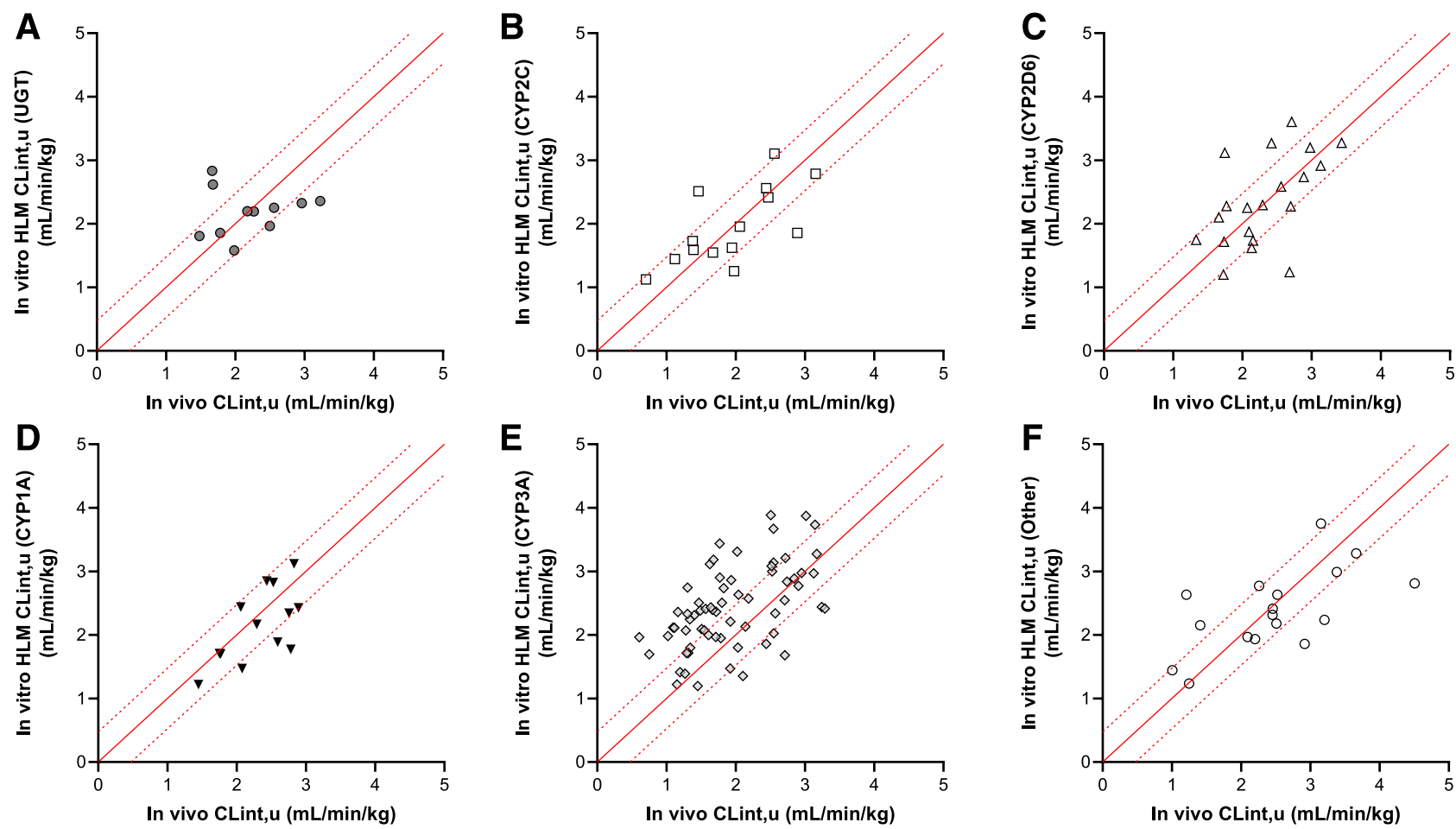

Fig. 4. IVIVE with a regression offset using HLM for substrates of drug metabolism enzyme families. (A) UGT, (B) CYP2C, (C) CYP2D6, (D) CYP1A, (E) CYP3A, and (F) Other. Where, the red solid line is the line of unity, and the red dotted lines represent a 3 -fold difference.

Furthermore, based on the data obtained herein, it is reassuring that our current approach of employing an IVIVE regression offset from data generated using $\mathrm{HH}$ remains a useful and applicable strategy for all compounds, irrespective of metabolism enzyme. However, in the authors' opinion, uncertainty in CLmet prediction would increase for compounds that were relatively unstable in HLM compared with a more favorable HH CLint value, which highlights the need to use both HLM and HH CLint data to optimize against in drug discovery.
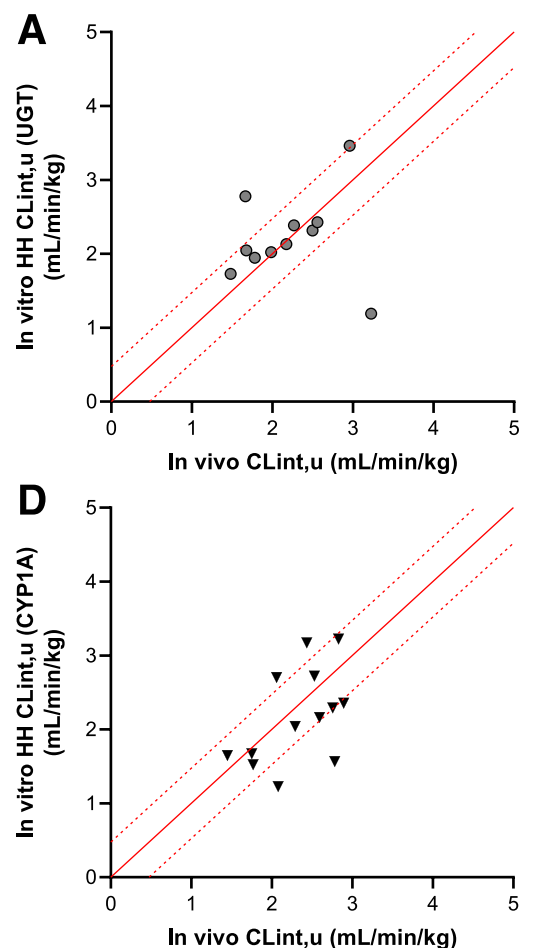



Fig. 5. IVIVE with a regression offset using HH for substrates of drug metabolism enzyme families. (A) UGT, (B) CYP2C, (C) CYP2D6, (D) CYP1A, (E) CYP3A, and (F) Other. Where, the red solid line is the line of unity, and the red dotted lines represent a 3-fold difference. 
TABLE 2

IVIVE prediction accuracy with and without a regression offset for CYP3A and non-CYP3A substrates

\begin{tabular}{|c|c|c|c|c|c|c|c|}
\hline & \multirow{2}{*}{ Substrates } & \multirow{2}{*}{$\begin{array}{l}\text { IVIVE } \\
\text { Approach }\end{array}$} & \multirow{2}{*}{ AFE } & \multirow{2}{*}{ AAFE } & \multicolumn{3}{|c|}{ IVIVE Prediction (\%) } \\
\hline & & & & & Over & Correct & Under \\
\hline \multirow[t]{3}{*}{ HLM } & \multirow[t]{2}{*}{ CYP3A } & Offset & 3.1 & 4.8 & 56 & 33 & 11 \\
\hline & & No Offset & 1.1 & 3.2 & 20 & 61 & 19 \\
\hline & Non-CYP3A & No Offset & 0.3 & 4.4 & 4 & 34 & 62 \\
\hline \multirow[t]{2}{*}{$\mathrm{HH}$} & \multirow[t]{2}{*}{ CYP3A } & Offset & 1.6 & 3.1 & 27 & 62 & 11 \\
\hline & & No Offset & 0.5 & 3.2 & 13 & 54 & 33 \\
\hline
\end{tabular}

Underprediction of CL could have detrimental effects on the candidate drug progression and may even result in the termination of the clinical program because of insufficient exposure to test the clinical hypothesis. Hence, in drug discovery, it remains an appropriate if risk-averse strategy to focus on lowering HLM in addition to HH CLint to maximize intrinsic metabolic stability. Thus the authors' recommendation remains to progress candidate drugs into the clinic with a suitable $\mathrm{HH}$ derived CLmet prediction and a correspondingly low HLM CLint. In addition, because a different IVIVE regression offset is now emerging for CYP3A substrates using HLM, the relationship may begin to elucidate a more comprehensive mechanistic understanding of IVIVE rather than the current empirical approach, which, although it is broadly successful, can be improved upon.
In summary, this work highlights the correlation between CYP3A substrates and the HLM:HH disconnect and the subsequent deleterious effect on the accuracy of metabolic CL predictions using HLM with our current IVIVE approach for this group of compounds. We demonstrated the HLM:HH CLint ratio is not correlated with MW, $\operatorname{LogD}$, pKa, human CL, or human Vd. However, more work is required to understand the association, if any, of efflux transporter activity with the HLM:HH disconnect. This work suggests a consistent IVIVE approach can be successfully applied to all compounds using $\mathrm{HH}$ irrespective of the main contributing metabolic enzyme and provides enhanced scaling methodologies using HLM. However, without a full mechanistic understanding of this HLM:HH disconnect phenomena, it remains our strategy to minimize both HLM as well as HH CLint.

\section{A \\ 要}



C





Fig. 6. IVIVE from HLM and HH. Prediction of in vivo CLint,u (A) using HLM with a regression offset, (B) using HLM without a regression offset, and (C) using $\mathrm{HH}$ with a regression offset. Where, the blue circles represent CYP3A substrates, large blue circles represent CYP3A substrates with an HLM:HH CLint ratio $>2$, and the red circles represent non-CYP3A substrates. The black solid line is the line of unity, and the black dotted lines represent a 3-fold difference. 


\section{Acknowledgments}

The authors would like to acknowledge Barry Jones, Tashinga Bapiro, Simone Stahl, Michael Davies, and Jolyon Faria for insightful scientific discussion throughout this work.

\section{Authorship Contributions}

Participated in research design: Williamson, Harlfinger, McGinnity.

Conducted experiments: Williamson.

Performed data analysis: Williamson.

Wrote or contributed to the writing of the manuscript: Williamson, Harlfinger, McGinnity.

\section{References}

Berezhkovskiy LM (2011) The corrected traditional equations for calculation of hepatic clearance that account for the difference in drug ionization in extracellular and intracellular tissue water and the corresponding corrected PBPK equation. J Pharm Sci 100:1167-1183.

Bowman CM and Benet LZ (2016) Hepatic clearance predictions from in vitro-in vivo extrapolation and the biopharmaceutics drug disposition classification system. Drug Metab Dispos 44 $1731-1735$.

Bowman CM and Benet LZ (2019a) In vitro-in vivo extrapolation and hepatic clearance-dependent underprediction. J Pharm Sci 108:2500-2504.

Bowman CM and Benet LZ (2019b) In vitro-in vivo inaccuracy: the CYP3A4 anomaly. Drug Metab Dispos 47:1368-1371.

Cerny MA (2016) Prevalence of non-cytochrome P450-mediated metabolism in Food and Drug Administration-approved oral and intravenous drugs: 2006-2015. Drug Metab Dispos 44: 1246-1252.

Chen S, Prieto Garcia L, Bergström F, Nordell P, and Grime K (2017) Intrinsic clearance assay incubational binding: a method comparison. Drug Metab Dispos 45:342-345.

Chiba M, Ishii Y, and Sugiyama Y (2009) Prediction of hepatic clearance in human from in vitro data for successful drug development. AAPS J 11:262-276.

Davies M, Jones RDO, Grime K, Jansson-Löfmark R, Fretland AJ, Winiwarter S, Morgan P, and McGinnity DF (2020) Improving the accuracy of predicted human pharmacokinetics: lessons learned from the AstraZeneca drug pipeline over two decades. Trends Pharmacol Sci 41 390-408.

Di L, Keefer C, Scott DO, Strelevitz TJ, Chang G, Bi YA, Lai Y, Duckworth J, Fenner K, Troutman MD, et al. (2012) Mechanistic insights from comparing intrinsic clearance values between human liver microsomes and hepatocytes to guide drug design. Eur J Med Chem 57: 441-448, doi: 10.1016/j.ejmech.2012.06.043 In press.

Dinno A (2015) Nonparametric pairwise multiple comparisons in independent groups using Dunn's test. Stata J 15:292-300.

Foster JA, Houston JB, and Hallifax D (2011) Comparison of intrinsic clearances in human liver microsomes and suspended hepatocytes from the same donor livers: clearancedependent relationship and implications for prediction of in vivo clearance. Xenobiotica 41 $124-136$
Hay M, Thomas DW, Craighead JL, Economides C, and Rosenthal J (2014) Clinical development success rates for investigational drugs. Nat Biotechnol 32:40-51.

Huang L, Berry L, Ganga S, Janosky B, Chen A, Roberts J, Colletti AE, and Lin MH (2010) Relationship between passive permeability, efflux, and predictability of clearance from in vitro metabolic intrinsic clearance. Drug Metab Dispos 38:223-231.

Kim RB, Wandel C, Leake B, Cvetkovic M, Fromm MF, Dempsey PJ, Roden MM, Belas F, Chaudhary AK, Roden DM, et al. (1999) Interrelationship between substrates and inhibitors of human CYP3A and P-glycoprotein. Pharm Res 16:408-414.

Lombardo F, Berellini G, and Obach RS (2018) Trend analysis of a database of intravenous pharmacokinetic parameters in humans for 1352 drug compounds. Drug Metab Dispos 46 $1466-1477$.

Mcdonald JH (2014a) Handbook of Biological Statistics, 3rd ed pp 157-164, Sparky House Publishing, Baltimore, MD

Mcdonald JH (2014b) Handbook of Biological Statistics, 3rd ed pp 186-189, Sparky House Publishing, Baltimore, MD.

Riley RJ, McGinnity DF, and Austin RP (2005) A unified model for predicting human hepatic, metabolic clearance from in vitro intrinsic clearance data in hepatocytes and microsomes. Drug Metab Dispos 33:1304-1311.

Rowland M, Benet LZ, and Graham GG (1973) Clearance concepts in pharmacokinetics. J Pharmacokinet Biopharm 1:123-136.

Shebley M, Liu J, Kavetskaia O, Sydor J, de Morais SM, Fischer V, Nijsen MJMA, and Bow DAJ (2017) Mechanisms and predictions of drug-drug interactions of the hepatitis $C$ virus three directacting antiviral regimen: paritaprevir/ritonavir, ombitasvir, and dasabuvir. Drug Metab Dispos 45:755-764.

Sohlenius-Sternbeck AK, Jones C, Ferguson D, Middleton BJ, Projean D, Floby E, Bylund J, and Afzelius L (2012) Practical use of the regression offset approach for the prediction of in vivo intrinsic clearance from hepatocytes. Xenobiotica 42:841-853.

Stringer R, Nicklin PL, and Houston JB (2008) Reliability of human cryopreserved hepatocytes and liver microsomes as in vitro systems to predict metabolic clearance. Xenobiotica 38:1313-1329. Weaver KF, Morales VC, Dunn SL, Godde K, and Weaver PF (2017) Kruskal-Wallis, An In troduction to Statistical Analysis in Research pp 353-391, John Wiley \& Sons Inc., Hoboken, NJ.

Williamson B, Colclough N, Fretland AJ, Jones BC, Jones RDO, and McGinnity DF (2020) Further considerations towards an effective and efficient oncology drug discovery DMPK strategy. Curr Drug Metab 21:145-162.

Winiwarter S, Chang G, Desai P, Menzel K, Faller B, Arimoto R, Keefer C, and Broccatell F (2019) Prediction of fraction unbound in microsomal and hepatocyte incubations: a comparison of methods across industry datasets. Mol Pharm 16:4077-4085.

Wood FL, Houston JB, and Hallifax D (2017) Clearance prediction methodology needs fundamental improvement: trends common to rat and human hepatocytes/microsomes and implications for experimental methodology. Drug Metab Dispos 45:1178-1188.

Yang J, Jamei M, Yeo KR, Rostami-Hodjegan A, and Tucker GT (2007) Misuse of the well-stirred model of hepatic drug clearance. Drug Metab Dispos 35:501-502.

Address correspondence to: Beth Williamson, AstraZeneca, Hodgkin Bldg., c/o Darwin Bldg., Unit 310, Cambridge Science Park, Milton Rd., Cambridge CB4 oWG, UK. E-mail: beth.williamson1@astrazeneca.com 